\title{
Neue Wege für den Ausbau schriftsprachlicher Fähigkeiten von herkunftssprachlichen LernerInnen im schulischen Russischunterricht
}

Jule Böhmer (Hamburg)

Преподавание русского языка как иностранного (РКИ) в Германии вызывает на данный момент, пожалуй, больше затруднений в области АиАактики, чем Аюбой Аругой иностранный язык. САожность заключается в том, что во многих школах классы очень гетерогенные, т.е. они состоят из учащихся, Аля которых русский язык явАяется иностранным, а также тех, Аля кого этот язык - родной. В связи с этим в Аанной статье рассматриваются возможности развития письменной речи на уроках РКИ у учащихся, Аля которых русский явАяется родным (семейным или унаследованным). В статье представлен дидактико-методический концепт, разработанный на основе теоретических предположений, актуальных результатов исследований и практических наблюдений. Основанный на Аифференцированном подходе метод способствует развитию письменной речи у носителей русского языка как родного.

\section{Theoretische Grundlagen}

Dieser Beitrag hat das Ziel, didaktisch-methodische Vorschläge für den Ausbau schriftlicher Fähigkeiten in der Herkunftssprache Russisch vorzulegen. Zunächst sollen an dieser Stelle theoretische Grundlagen und Forschungsergebnisse vorgestellt werden, auf denen meine späteren Ausführungen aufbauen.

Seit 2001 haben unterschiedliche Schulleistungsstudien immer wieder die Bedeutung bildungssprachlicher Fähigkeiten für den Schulerfolg untermauert (Baumert, Stanat \& Watermann 2006, Bos et al. 2007). Interessant für den vorliegenden Beitrag sind aber v. a. Studien, die zeigen, dass bilinguale SchülerInnen, 
die in ihrer Herkunftssprache alphabetisiert sind, über bessere schulfremdsprachliche Fähigkeiten verfügen als ihre monolingualen MitschülerInnen. Bilinguale SchülerInnen, die ihre Herkunftssprache weder lesen noch schreiben können, liegen mit ihren Ergebnissen zwischen den beiden erstgenannten Gruppen (vgl. u. a. Rauch, Jurecka \& Hesse 2010).

Für die Beschäftigung mit Bilingualität und Biliteralität stellt die Annahme der Interdependenzhypothese eine nützliche Grundlage dar (Cummins 1979). Ausgehend von dieser Hypothese wird angenommen, dass metasprachliches Wissen von einer L1 auf eine L2 übertragen wird (Cummins 2010: 15). Diese Annahme bildet die Grundlage für die didaktisch-methodische Ausrichtung herkunftssprachlichen Unterrichts in Deutschland und für das Konzept der durchgängigen Sprachbildung (Gogolin \& Lange 2010).

Studien mit herkunftssprachlichen SchülerInnen haben gezeigt, dass es nicht den/die HerkunftssprecherIn mit einer bestimmten Ausprägung sprachlicher Fähigkeiten gibt, sondern vielmehr eine Gruppe von HerkunftssprecherInnen, die in Hinblick auf ihre sprachlichen Fähigkeiten stark heterogen ist. Verschiedene soziolinguistische Einflussfaktoren splitten die Gruppe in viele Subgruppen auf mit einer großen Spannbreite herkunftssprachlicher Fähigkeiten (von rudimentär rezeptiven bis hin zu sehr guten produktiven Fähigkeiten) (vgl. Polinsky \& Kagan 2007). Diese große Spannbreite ist auch unter den herkunftssprachlichen LernerInnen im schulischen Russischunterricht zu finden (vgl. Mehlhorn 2016). Erste Ergebnisse aus dem bisher noch nicht weit verbreiteten Gebiet der Biliteralitätsforschung zeigen, dass die Förderung der herkunftssprachlichen Schreib- und Lesefähigkeiten positive Effekte auf literate Fähigkeiten im Deutschen hervorbringt (Böhmer 2015, Roll, Gürsoy \& Boubakri 2016, Yilmaz Woerfel \& Riehl 2016).

Die Auseinandersetzung mit der Fertigkeit Schreiben basiert hier auf dem Schreibmodell von Hayes \& Flower (1980), welches 2001 um den für den vorliegenden Beitrag relevanten Aspekt des Schreibens in der L2 erweitert wurde. Grießhabers (2001, vgl. auch Wolff 1992) Ergänzungen visualisieren die Schwierigkeiten von L2 SchreiberInnen, die auf empirischen Beobachtungen basieren. Sie zeigen, dass mangelndes idiomatisches Wissen sowie lexikalische und syntaktische Unsicherheiten den Schreibprozess in der L2 behindern können und dass eine längere Planungszeit und mehr Zeit zum Schreiben benötigt werden.

Anka Bergmann, Olga Caspers \& Wolfgang Stadler (Hg.)

Didaktik der slawischen Sprachen - Beiträge zum 1. Arbeitskreis in Berlin (12.-14.9.2016)

(C) 2018 innsbruck university press, ISBN 978-3-903187-11-5, DOI 10.15203/3187-11-5 
Aus diesem Grund sind Texte in der L2 häufig durch einen geringeren Umfang gekennzeichnet, basieren auf einem begrenzten Wortschatz sowie einfachen Satzstrukturen und beinhalten grammatische Abweichungen (vgl. Kristmanson, Dicks \& Le Bouthillier 2009).

Neue schreibdidaktische Ansätze stützen sich auf das textprozedurenorientierte Modell von Bachmann \& Feilke (2014). Demnach müssen bei der Vermittlung und Entwicklung von schriftsprachlichen Fähigkeiten zwei unterschiedliche Ebenen berücksichtigt werden: einerseits die Schemaebene, auf der festgelegt ist, wie eine Textsorte aufgebaut ist und welche textstrukturellen Elemente sie zu dieser machen; andererseits die Ausdrucksebene, auf der sich sprachliche Mittel festlegen lassen, die für die Realisierung dieser Textsorte in einer bestimmten Sprache sorgen.

\section{Rahmenbedingungen des schulischen Russischunterrichts}

Begonnen werden soll mit einigen grundsätzlichen Überlegungen zu den Rahmenbedingungen, unter denen schulischer Russischunterricht in Deutschland zurzeit stattfindet. Dabei werden verschiedene Einflussfaktoren wie LernerInnen, Lehrmaterialien und didaktisch-methodische Ausrichtungen in den Blick genommen:

- Unabhängig von der Organisationsform (Fremdsprachen- oder Herkunftssprachenunterricht ${ }^{1}$ ), ist die SchülerInnenschaft, zumindest in den (westdeutschen) Ballungsräumen, sprachlich sehr heterogen. FremdsprachenlernerInnen und herkunftssprachliche LernerInnen mit unterschiedlichen Voraussetzungen lernen gemeinsam. Im kommunikativ ausgerichteten Fremdsprachenunterricht haben die herkunftssprachlichen LernerInnen besonders im Bereich mündlicher Fertigkeiten Lernvorteile. Grund da-

1 Herkunftssprachlichen Unterricht (u. a. für Russisch) als Angebot staatlicher Schulen gibt es in den Bundesländern Bremen, Niedersachsen, Hamburg, Nordrhein-Westfalen, Rheinland-Pfalz, Hessen und Saarland (vgl. Böhmer 2016).

Anka Bergmann, Olga Caspers \& Wolfgang Stadler (Hg.)

Didaktik der slawischen Sprachen - Beiträge zum 1. Arbeitskreis in Berlin (12.-14.9.2016)

(C) 2018 innsbruck university press, ISBN 978-3-903187-11-5, DOI 10.15203/3187-11-5 
für ist, dass die mündlichen, alltagssprachlichen Fähigkeiten bei ihnen (meist) besser ausgeprägt sind als das schriftsprachliche Register. In Hamburg wird beobachtet, dass, falls herkunftssprachliche SprecherInnen eine private russische Schule in ihrer Freizeit besuchen, der Besuch meist mit Beginn der Pubertät beendet wird.

- Bisher gibt es auf dem deutschen Markt nur wenige Lehrmaterialien, die auf die besonderen Bedürfnisse herkunftssprachlicher LernerInnen zugeschnitten sind. Daher wird an den meisten Schulen noch immer mit Lehrwerken für Russisch als zweite/dritte Fremdsprache gearbeitet. Für herkunftssprachliche LernerInnen öffnet sich somit ein Spannungsfeld zwischen (für FremdsprachenlernerInnen konzipierten) Lehrwerksinhalten, Altersangemessenheit und verfügbaren Sprachfähigkeiten. Der Forderung nach Differenzierung kann im Schulalltag häufig aufgrund von fehlender Zeit und unzureichendem passenden Material nicht nachgekommen werden.

- Der fehlende Einsatz von interdisziplinären und fächerübergreifenden Arbeitsphasen in Schulen bedingt, dass SchülerInnen nicht über Fächergrenzen hinweg denken lernen. Häufig können sie deshalb erworbenes Wissen über Fächergrenzen hinweg nicht anwenden und entsprechende Transferleistungen nicht erbringen.

- Eine Folge der Kompetenzorientierung in der schulischen Bildungslandschaft ist eine starke Textsortenorientierung des Deutschunterrichts in Sekundarstufe I und II. In diesem textsortenbasierten Unterricht werden die Charakteristika unterschiedlicher Textsorten sehr detailliert und ausführlich besprochen. Zudem werden - wie in allen anderen Fächern auch - Operatoren zur Vereinheitlichung von Aufgabenstellungen eingeführt (s. z. B. Freie und Hansestadt Hamburg, Behörde für Schule und Berufsbildung 2016). Der Fokus auf den Ausbau der schriftsprachlichen Fähigkeiten durch die Förderung von Textsortenkompetenz schlägt sich auch in den Rahmenplänen für die einzelnen Fächer nieder (z. B. Freie und Hansestadt Hamburg, Behörde für Schule und Berufsbildung 2011). 


\section{Didaktisch-methodische Konzeption zur Schriftsprach- entwicklung}

Die im Folgenden vorgestellte didaktisch-methodische Konzeption zur Schriftsprachentwicklung basiert - wie schon erwähnt - auf dem textprozedurenorientierten Modell von Bachmann \& Feilke (2014). Aus diesem lassen sich didaktische Handlungsanweisungen zur systematischen Schreibförderung ableiten. Indem die Textsortenkompetenz, die die SchülerInnen im Deutschunterricht erwerben, im Russischunterricht aufgegriffen wird und sprachliche Mittel zur Realisierung im Russischen erarbeitet werden, können die schriftsprachlichen Fähigkeiten im Russischen gezielt ausgebaut werden. Dabei soll die entwickelte Konzeption zur Schriftsprachentwicklung als Differenzierungsangebot für herkunftssprachliche SchülerInnen einsetzbar sein. Aus diesem Grund nützt der folgende Ansatz zum Ausbau der Schriftsprache ein Lehrwerk für den fremdsprachlichen Russischunterricht. In den folgenden Abschnitten 3.1 und 3.2 wird auf das Lehrwerk Конечно! (Russisch als zweite Fremdsprache; Bände 2 und 3) (Amstein-Bahmann et al. 2010) für die Klassenstufen $7 / 8$ zurückgegriffen. Die dargelegten Ausführungen sind beispielhaft und können in Abhängigkeit vom verwendeten Lehrwerk, der Klassenstufe und der im Deutschunterricht entwickelten Textsortenkompetenz modifiziert werden.

\subsection{Bezug zum Deutschunterricht}

Der Fokus, der im Deutschunterricht auf die Entwicklung der Textsortenkompetenz gelegt wird, soll genutzt werden, um die schriftsprachlichen Fähigkeiten von herkunftssprachlichen LernerInnen im Russischen auszubauen. Die im Deutschunterricht besprochenen Textsorten werden im Russischunterricht aufgegriffen. Die SchülerInnen kennen auf der Schemaebene bereits jene Strukturen, die die jeweilige Textsorte charakterisieren, und lernen dann im Russischunterricht für die Ausdrucksebene sprachliche Mittel kennen, die sie befähigen, diese Textsorte auch im Russischen zu realisieren. Da in der gymnasialen Oberstufe in allen Fremdsprachen der rezeptive und produktive Umgang mit den aus dem Deutsch-

Anka Bergmann, Olga Caspers \& Wolfgang Stadler (Hg.)

Didaktik der slawischen Sprachen - Beiträge zum 1. Arbeitskreis in Berlin (12.-14.9.2016)

(C) 2018 innsbruck university press, ISBN 978-3-903187-11-5, DOI 10.15203/3187-11-5 
unterricht bekannten Textsorten gefordert wird, werden Lerninhalte aus späteren Lernjahren für die herkunftsprachlichen SchülerInnen vorgezogen.

$\mathrm{Da}$ mit fortschreitender Bildungsbiographie die sachlich-argumentativen Textsorten an Bedeutung gewinnen, stehen sie in diesem Beitrag im Mittelpunkt. Zunächst werden anhand von Rahmenplan und Lehrwerken die wichtigsten sachlich-argumentativen Textsorten in den Klassenstufen 7/8 herausgearbeitet (s. Tab. 1) $)^{2}$.

Tabelle 1: Sachlich-argumentative Textsorten in den Stufen 7/8

\begin{tabular}{|c|c|c|}
\hline \multicolumn{3}{|c|}{ Klassenstufe 7} \\
\hline Stellungnahme & Beschreibung & Inhaltsangabe \\
\hline $\begin{array}{l}\text { - Darstellung eines } \\
\text { eigenen Standpunktes } \\
\text { - Grundlagen der Argu- } \\
\text { mentation bilden } \\
\text { Fakten, gemeinsame } \\
\text { Werte (verdeutlicht } \\
\text { anhand von Beispielen) } \\
\text { - evtl. Entkräften von } \\
\text { Gegenargumenten } \\
\text { - Adressatenbezogenheit } \\
\text { - Einleitung: Darstellung } \\
\text { der „Streitfrage“/des } \\
\text { „Problems“ } \\
\text { - Hauptteil: Anführen der } \\
\text { Argumente in sinnvoller } \\
\text { Reihenfolge } \\
\text { - Schluss: mit Fazit }\end{array}$ & $\begin{array}{l}\text { - Sachliches Informieren } \\
\text { - Klarheit (nachvollzieh- } \\
\text { bare Reihenfolge) } \\
\text { - Anschaulichkeit } \\
\text { (Sachstil) } \\
\text { - Genauigkeit } \\
\text { - Neutralität } \\
\text { - Adressatenbezug } \\
\text { - Bildbeschreibung: } \\
\text { KünstlerIn, Art der } \\
\text { Darstellung, Thema, } \\
\text { wichtige Elemente }\end{array}$ & $\begin{array}{l}\text { - nüchtern/sachlich } \\
\text { - Präsens } \\
\text { - kurz } \\
\text { - Einleitung: Textsorte, } \\
\text { Titel, Autor, Erschei- } \\
\text { nungsjahr, Thema } \\
\text { - Hauptteil: Wieder- } \\
\text { gabe des Geschehens } \\
\text { in knapper Form } \\
\text { (alles Wichtige } \\
\text { eingeschlossen) } \\
\text { - Schluss: Fazit }\end{array}$ \\
\hline
\end{tabular}

2 Die Analyse wurde anhand des Hamburger Rahmenplans für das Fach Deutsch in der Sekundarstufe I und entsprechender Lehrwerke durchgeführt. Die Inhalte stimmen mit denen anderer Bundesländer überein. 


\begin{tabular}{|l|l|l|}
\hline \multicolumn{3}{|c|}{ Klassenstufe 8 } \\
\hline $\begin{array}{c}\text { Personenbeschreibung/ } \\
\text { Charakterisierung }\end{array}$ & $\begin{array}{c}\text { Interpretation von } \\
\text { Prosa/Lyrik }\end{array}$ & \multicolumn{1}{|c|}{ Erörterung } \\
\hline - präzise & - Einleitung: Titel, Autor, \\
- sehr genaue und detail- & Textsorte, Inhalt/Thema \\
lierte Darstellung der & mit kurzer Inhaltsangabe & Einleitung: \\
klare Nennung des & Problems/Themas \\
äußeren Merkmale einer & - Hauptteil: Beschreibung, & - Hauptteil: Darstel- \\
Fuß) & Erklärung, Deutung & lung von Pro- \& \\
- Präsens & wesentlicher Inhalts- und & Kontraargumenten \\
- (Charakterisierung: & Gestaltungselemente, & (nach Gewichtung) \\
Beschreibung der & Zusammenfassung & - Schluss: Fazit/ \\
äußeren und inneren & wesentlicher Ergebnisse & abschließende \\
Situation der Person, & - Schluss: abschließende & Forderung \\
u. a. Charaktereigen- & Beurteilung der & \\
schaften, Entwicklung & Geschichte nach Sinn & \\
der Person) & und Gestaltung & \\
\hline
\end{tabular}

Tab. 1 zeigt, welche sachlich-argumentativen Texte in den Stufen 7/8 im Deutschunterricht behandelt werden und über welche besonderen Merkmale sie auf der Schemaebene verfügen.

\subsection{Vorschlag für ein Schreibcurriculum}

Um auf die aus dem Deutschunterricht bekannten Textsorten im Russischunterricht Bezug nehmen zu können, werden Möglichkeiten gesucht, wie sich diese Textsorten mit den Inhalten des verwendeten Lehrwerks im Russischunterricht verknüpfen lassen.

Die kommunikative Ausrichtung des modernen Fremdsprachenunterrichts spiegelt sich im Aufbau der Lehrwerke wider. Eine Lehrwerkseinheit basiert auf einer neuen zu bewältigenden Kommunikationssituation, die dann die Einführung einer neuen grammatischen Struktur oder bestimmter sprachlicher Mittel evoziert. Zur Entwicklung von Differenzierungsmaßnahmen für herkunfts- 
sprachliche LernerInnen werden nun einzelne Lektionen aus dem Lehrwerk Конечно! (Amstein-Bahmann et al. 2010) mit den o. g. Textsorten verknüpft. Für die Verknüpfung werden die kommunikativen Ziele einer Lehrwerkslektion mit den Textsorten in Bezug auf Inhalt und Lexik abgeglichen und verbunden. Im Sinne eines spiralcurricularen Aufbaus wird folgender Vorschlag (Tab. 2) für ein Schreibcurriculum für herkunftssprachliche LernerInnen erarbeitet.

Tabelle 2: Schreibcurriulum für herkunftssprachliche LernerInnen in den Klassenstufen 7/8

\begin{tabular}{|c|c|c|c|}
\hline \multicolumn{4}{|c|}{ Klassenstufe 7} \\
\hline $\begin{array}{l}\text { Lehrwerks- } \\
\text { lektion/ } \\
\text { Kommunika- } \\
\text { tionsziel }\end{array}$ & $\begin{array}{c}\text { Урок } 2 \text { «В Санкт- } \\
\text { Петербурге» } \\
\text { „sagen, dass man etwas } \\
\text { will/nicht will, den } \\
\text { Grund angeben“ }\end{array}$ & $\begin{array}{c}\text { Урок } 4 \text { «Вечеринка у } \\
\Lambda \text { ены» } \\
\text { „ein Zimmer } \\
\text { beschreiben“ }\end{array}$ & $\begin{array}{c}\text { Урок } 5 \text { «ГАе мы } \\
\text { учимся»* }\end{array}$ \\
\hline Schemaebene & Stellungnahme & Beschreibung & Inhaltsangabe \\
\hline $\begin{array}{l}\text { Ausdrucks- } \\
\text { ebene }\end{array}$ & $\begin{array}{l}\text { - Textgliederung I } \\
\text { - eigene Meinung } \\
\text { ausdrücken I }\end{array}$ & $\begin{array}{l}\text { - Satzverbindungen/ } \\
\text { Konnektoren }\end{array}$ & $\begin{array}{l}\text { - Satzbausteine für } \\
\text { Einleitung }\end{array}$ \\
\hline \multicolumn{4}{|c|}{ Klassenstufe 8} \\
\hline $\begin{array}{l}\text { Lehrwerks- } \\
\text { lektion/ } \\
\text { Kommunika- } \\
\text { tionsziel }\end{array}$ & $\begin{array}{c}\text { Урок 1 } \\
\text { «Внешность или } \\
\text { характер?» } \\
\text { „Personen beschreiben } \\
\text { und charakterisieren“ }\end{array}$ & $\begin{array}{c}\text { Урок } 3 / 4 \\
\text { «По обмену в } \\
\text { России»/ «Мы умеем } \\
\text { веселиться»* }\end{array}$ & $\begin{array}{c}\text { Урок } 5 \\
\text { «Международный } \\
\text { магерь 'Чистый } \\
\text { берег'» } \\
\text { „argumentieren und } \\
\text { die eigene Meinung } \\
\text { begründen“ }\end{array}$ \\
\hline Schemaebene & $\begin{array}{l}\text { Personenbeschreibung/ } \\
\text { Charakterisierung }\end{array}$ & $\begin{array}{l}\text { Interpretation von } \\
\text { Prosa/Lyrik }\end{array}$ & Erörterung \\
\hline $\begin{array}{l}\text { Ausdrucks- } \\
\text { ebene }\end{array}$ & $\begin{array}{l}\text { - Adjektive für Person- } \\
\text { enbeschreibung/- } \\
\text { charakterisierung }\end{array}$ & $\begin{array}{l}\text { - Fachtermini für } \\
\text { Interpretation }\end{array}$ & $\begin{array}{l}\text { - eigene Meinung } \\
\text { ausdrücken II } \\
\text { - Textgliederung II }\end{array}$ \\
\hline
\end{tabular}

* Zur Bearbeitung der Textsorten „Inhaltsangabe“ und „Interpretation“ müssen thematisch passende Texte hinzugezogen werden. 
Da die Inhalte der Schemaebene den SchülerInnen aus dem Deutschunterricht bekannt sein sollten, müssen diese nur wiederholt werden. Die Ausdrucksebene steht folglich im Fokus, neben der Wortschatzerweiterung kann die Arbeit am Text (z. B. Aufbau, Schaffung von Textkohärenz) ins Auge gefasst werden.

\section{$4 \quad$ Didaktisch-methodische Umsetzung}

Wie die konkrete didaktisch-methodische Umsetzung des Ausbaus schriftsprachlicher Fähigkeiten erfolgen kann, wird in 4.1 vorgestellt. Bei der Entwicklung von Unterrichtssequenzen ist darauf zu achten, dass auf das Wissen über Schemata der jeweiligen Textsorte zurückgegriffen wird und zum Ausbau der Ausdrucksmöglichkeiten auf Wort- und Satzebene Zusatz-/Erweiterungsmöglichkeiten angeboten werden. Im Sinne eines Spiralcurriculums sollten die sprachlichen Mittel zeitversetzt wiederholt und erweitert werden.

Der Ausbau der Textsortenkompetenz im Russischen kann nur als Differenzierungsangebot für herkunftssprachliche SchülerInnen erfolgen. Es bietet sich an, herkunftssprachliche SchülerInnen mit Aufgaben zum Ausbau der schriftlichen Textsortenkompetenz zu betrauen, während fremdsprachliche LernerInnen an mündlichen Kompetenzen arbeiten. Da herkunftssprachliche LernerInnen während dieser Unterrichtsphasen aufgrund ihrer Vorkenntnisse häufig unterfordert sind, können sie die Unterrichtszeit anderweitig sinnvoll nutzen.

Wie die konkrete Unterrichtsgestaltung aussieht, hängt von vielen Faktoren ab, wie Anzahl der fremd-/herkunftssprachlichen LernerInnen, Anzahl der zur Verfügung stehenden Stunden, räumliche und personelle Ressourcen etc. Die methodische Umsetzung (Erarbeitung der sprachlichen Mittel im Russischen, die Steuerung des Schreibprozesses) ist ebenso von diesen Faktoren abhängig.

\subsection{Beispiel für eine Unterrichtssequenz}

Im Folgenden wird ein Beispiel für eine Unterrichtssequenz vorgestellt, die die Textsorte „Beschreibung“ in den Blick nimmt. In Урок 4 „Вечеринка у Аенъг“des Lehrwerks Конечно! 2 (Amstein-Bahmann et al. 2010) lernen die Fremdsprachen-

Anka Bergmann, Olga Caspers \& Wolfgang Stadler (Hg.)

Didaktik der slawischen Sprachen - Beiträge zum 1. Arbeitskreis in Berlin (12.-14.9.2016)

(C) 2018 innsbruck university press, ISBN 978-3-903187-11-5, DOI 10.15203/3187-11-5 
lernenden Lexik aus dem Wortfeld „Wohnung" kennen und sollen als kommunikatives Ziel ein Zimmer beschreiben können. Anknüpfend daran kann für die herkunftssprachlichen LernerInnen an dieser Stelle die Textsorte „Beschreibung“ aufgegriffen werden.

\subsubsection{Rahmenbedingungen}

In der gewählten Kommunikationssituation aus Урок 4 „Вечеринка у Аенъ“ im Lehrwerk Конечно! 2 (Amstein-Bahmann et al. 2010) lädt die Protagonistin Lena ihre Freunde zu einer Party ein; diese sollen nach Eintreffen bei Lena eine Führung durch ihre Wohnung bekommen. Das kommunikative Ziel wird als „eine Wohnung/ein Zimmer beschreiben“ definiert. Dafür werden sprachliche Mittel aus dem Wortfeld квартира sowie Ortsangaben mithilfe von Präpositionen (рядам c, над, за, nод) und die Possessivpronomen im Singular neu eingeführt. Für diese Lektion liegt es nahe, die im Deutschunterricht in Klassenstufe 7 bearbeitete Textsorte „Beschreibung“ mit den herkunftssprachlichen SchülerInnen aufzugreifen und mit diesen die Anfertigung einer schriftlichen Beschreibung auf Russisch zu üben. Auf diese Weise können innerhalb des Lektionsthemas die schriftsprachlichen Fähigkeiten erweitert werden.

\subsubsection{Methodische Gestaltung}

Die didaktisch-methodische Gestaltung der Unterrichtssequenz erfolgt entlang des ECRI-Modells ${ }^{3}$. Diese fünfschrittige Abfolge sensibilisiert zunächst für die Charakteristika der Textsorte und begleitet anschließend den Schreibprozess:

Als Erstes wird den SchülerInnen ein Zielbeispieltext präsentiert, in diesem Fall eine Beschreibung eines Zimmers auf Russisch. Beispieltexte sind im Internet zu finden, da die Textsorte „описание“ auch in Russland in der Sekundarstufe I Unterrichtsinhalt ist.

3 Das ECRI-Modell wurde im kanadischen Schulkontext entwickelt. Die Voraussetzungen sind ähnlich: Bilinguale SchülerInnen, die in einer Sprache alphabetisiert worden sind, sollen schriftsprachliche Kenntnisse in ihrer L2 erwerben (Kristmanson et al. 2009).

Anka Bergmann, Olga Caspers \& Wolfgang Stadler (Hg.)

Didaktik der slawischen Sprachen - Beiträge zum 1. Arbeitskreis in Berlin (12.-14.9.2016)

(C) 2018 innsbruck university press, ISBN 978-3-903187-11-5, DOI 10.15203/3187-11-5 
Im zweiten Schritt analysieren die SchülerInnen den Text und erkennen auf der schematischen Ebene die charakteristischen Merkmale: sachlich-neutraler Stil, Genauigkeit, Zeitform „Präsens“. Auf diese Weise wiederholen die SchülerInnen anhand des Beispieltextes noch einmal die Merkmale der Textsorte und sie stellen fest, dass eine Beschreibung auch im Russischen über dieselben charakteristischen Textmerkmale verfügt. Diese schematischen Merkmale werden durch eine hohe Anzahl von Adjektiven und Präpositionen auf der Ausdrucksebene unterstützt. Zahlreiche Konnektoren leiten Nebensätze ein. Diese sprachlichen Mittel werden gemeinsam für das Russische erarbeitet (auch unter Zuhilfenahme des Deutschen) und können zusätzlich zu denen im Lehrwerk verwendet werden. Für die Beschreibung können u. a. folgende Adjektive (огромньий, узкий, широкий, высокий), Präроsitionen (около, среди, напротив, у) und Konnektoren (так как, чтобъъ) verwendet werden. In einer Übungsphase wäre hier die Sicherung der neuen sprachlichen Mittel in Form von gestaffelten Übungen (von geschlossenen bis offenen Übungen) sinnvoll.

Im dritten Schritt leitet die Lehrkraft die Anfertigung einer gemeinsamen Beschreibung eines Zimmers an. Es empfiehlt sich, dies an der Tafel oder am Smartboard zu machen. In diesem öffentlichen Schreibprozess fragt die Lehrkraft ggf. nach, fordert zur Fehlerkorrektur auf oder regt zur Verwendung von anderen sprachlichen Mitteln an. Dieses Vorgehen fördert die Perspektive der SchülerInnen, Schreiben als Prozess zu sehen.

Im vierten Schritt verfassen die SchülerInnen eine Beschreibung eines weiteren fiktiven Zimmers in Partnerarbeit.

Im fünften Schritt stehen die SchülerInnen allein vor der Aufgabe, eine Beschreibung ihres Zimmers anzufertigen. Spätestens an dieser Stelle sollten die SchülerInnen mit dem Operator (hier: описать) vertraut gemacht werden, um eine mögliche Aufgabenstellung mit der geforderten Textsorte zu verknüpfen. Notwendig ist es, dabei auch den Imperativ des jeweiligen Operators (hier: опиши.) einzuführen und eine beispielhafte Aufgabenstellung (z. B. Опиии свою комнату!) zu zeigen. Vorteil bei diesem schrittweisen Vorgehen ist, dass die SchülerInnen langsam an die Textproduktion herangeführt werden und auf diese Weise Schreibroutinen entwickeln, welche helfen, die neu erworbenen lexikalischen und syntaktischen Strukturen auch im schriftsprachlichen Kontext anzuwenden.

Anka Bergmann, Olga Caspers \& Wolfgang Stadler (Hg.)

Didaktik der slawischen Sprachen - Beiträge zum 1. Arbeitskreis in Berlin (12.-14.9.2016)

(C) 2018 innsbruck university press, ISBN 978-3-903187-11-5, DOI 10.15203/3187-11-5 


\subsection{Weitere didaktisch-methodische Gestaltungsmöglichkeiten}

Neben der Präsentation einer Unterrichtssequenz nach dem ECRI-Modell lassen sich zahlreiche andere didaktisch-methodische Gestaltungsmöglichkeiten finden, von denen einige an dieser Stelle aufgezeigt werden.

Für die Texterstellung kann auf die Arbeit von Börner (1992) zurückgegriffen werden. Er entwarf für die drei Phasen des Schreibens (Planung, Formulierung und Überarbeitung) in der Fremdsprache sog. Schreibmaximen, welche von Gottwald (2016) für das Schreiben in der Herkunftssprache modifiziert wurden. Das Aufstellen solcher Schreibmaximen und ihr Einsatz bei der Textproduktion hilft herkunftssprachlichen SchülerInnen, ihre Texte besonders hinsichtlich Textstruktur und sprachlicher Gestaltung zu verbessern.

Für den Prozess der Textüberarbeitung lässt sich die Methode der Schreibkonferenz einsetzen, bei der die sprachliche Heterogenität der herkunftssprachlichen LernerInnen als Ressource genutzt wird. Ziel ist es, dass die verfassten Texte von den SchülerInnen gemeinsam überarbeitet und redigiert werden, sodass eine zufriedenstellende Endversion entsteht. Die von der Lehrperson angeleitete Analyse, sowie die anschließenden Korrektur- und Überarbeitungsschleifen der SchülerInnentexte durch die MitschülerInnen verdeutlichen die Prozesshaftigkeit der Texterstellung. Fortgeschrittene herkunftssprachliche LernerInnen unterstützen ihre MitschülerInnen bei der Korrektur und Verbesserung.

Für eine inhaltliche Differenzierung bietet sich eine in Abhängigkeit vom Sprachstand der herkunftssprachlichen SchülerInnen andere (weit über das Lehrwerk hinausgehende) lexikalische und inhaltliche Schwerpunktsetzung an. So können die neu eingeführten sprachlichen Mittel beliebig erweitert werden und auch die inhaltlich-thematische Ausgestaltung kann weit über die Lehrwerksinhalte hinausgehen. Besonders für fortgeschrittene herkunftssprachliche LernerInnen bietet sich der Rückgriff auf Methoden des Deutschunterrichts an, die die Textproduktion in den Mittelpunkt stellen (z. B. Anfertigung von Kommentaren, Rezensionen, Interpretationen).

Eine zusätzliche inhaltliche und sprachliche Erweiterungsmöglichkeit bietet die Aufnahme von Textsorten aus anderen Fächern (z. B. Protokolle aus dem Physik- oder Quellenanalysen aus dem Geschichtsunterricht). Erste Ergebnis-

Anka Bergmann, Olga Caspers \& Wolfgang Stadler (Hg.)

Didaktik der slawischen Sprachen - Beiträge zum 1. Arbeitskreis in Berlin (12.-14.9.2016)

(C) 2018 innsbruck university press, ISBN 978-3-903187-11-5, DOI 10.15203/3187-11-5 
se einer Studie, in der Textsortenkompetenz im Herkunftssprachen- und Sach-/ Fachunterricht koordiniert entwickelt wurde, zeigen, dass sich die Förderung mehrsprachiger literaler Fähigkeiten positiv auf die gesamten sprachlichen Fähigkeiten auswirkt (vgl. Roll \& Beese 2015). Dieses Vorgehen bietet viele Erweiterungsmöglichkeiten und fördert die individuelle mehrsprachige Literalität.

\section{$5 \quad$ Fazit und Ausblick}

Fremdsprachliche und herkunftssprachliche LernerInnen mit unterschiedlichen Vorkenntnissen stellen RussischlehrerInnen vor große didaktische Herausforderungen. Denn beide LernerInnengruppen bedürfen einer unterschiedlichen didaktisch-methodischen Herangehensweise. In Ermangelung an spezifischen Lehrwerken für herkunftssprachliche SchülerInnen wird in Deutschland meist auf Lehrwerke für den fremdsprachlichen Unterricht zurückgegriffen. Damit können die Bedürfnisse von herkunftssprachlichen LernerInnen, die zumeist im Bereich der schriftsprachlichen Fähigkeiten liegen, nicht ausreichend berücksichtigt werden.

Im vorliegenden Beitrag wurde ein Ansatz vorgestellt, wie die schriftsprachlichen Fähigkeiten von herkunftssprachlichen SchülerInnen verbessert werden können. Ausgehend von der Beobachtung, dass herkunftssprachliche SchülerInnen im fremdsprachlich ausgerichteten Russischunterricht in vielen Unterrichtsphasen unterfordert sind, wurde nach Maßnahmen gesucht, wie herkunftssprachliche LernerInnen nach ihren Möglichkeiten bestmöglich gefördert werden können. Im Sinne der effizienten Materialentwicklung wurde ein didaktisch-methodischer Ansatz präsentiert, der auf Grundlage eines Lehrwerks für den fremdsprachlichen Russischunterricht eine Differenzierungsmöglichkeit bietet. Hierfür wird auf die Textsortenkompetenz der SchülerInnen aus dem Deutschunterricht zurückgegriffen, mithilfe derer die schriftsprachlichen Fähigkeiten weiterentwickelt werden. Somit wird interdisziplinäres Arbeiten zum Ausbau der Zweisprachigkeit genutzt. Der Sprachausbau erfolgt im Russischen v. a. auf lexikalischer und syntaktischer Ebene im schriftsprachlichen Register. 
Der hier präsentierte Ansatz lässt sich modifizieren und ergänzen. Die vorgestellte Gestaltung nach dem ECRI-Modell ist eine Möglichkeit, allerdings lassen sich auch andere Methoden aus der Schreibdidaktik (z. B. Schreibkonferenzen) anwenden: Je nach Sprachstand der herkunftssprachlichen SchülerInnen bieten sich weitere Differenzierungsmöglichkeiten durch die Steuerung zusätzlicher sprachlicher Mittel bzw. die thematische Erweiterung an. Ebenso können Textsorten aus anderen Unterrichtsfächern (z. B. Protokolle aus den naturwissenschaftlichen Fächern) besprochen und angefertigt werden.

Sinnvoll wäre es, entlang der sachlich-argumentativen Textsorten des (Deutsch-)Unterrichts ein Schreibcurriculum für herkunftssprachliche SchülerInnen für die Sekundarstufe I zu entwickeln, das spiralcurricular aufgebaut ist und auf diese Weise die lexikalischen und syntaktischen sprachlichen Mittel stetig erweitert. Durch eine engere Zusammenarbeit der LehrerInnen für die jeweiligen Fächer könnten die Lernprozesse effektiver gestaltet werden.

Für die Zukunft wäre es wünschenswert, wenn die vorgestellte didaktisch-methodische Konzeption der Schriftsprachentwicklung zur Verzahnung von fremd-/ herkunftssprachlichen Unterrichtsangeboten und Sachfächern etabliert würde. Auf diese Weise wäre die Förderung individueller mehrsprachiger Literalität ein Teil durchgängiger Sprachbildung an Schulen, die allen SchülerInnen - im Sinne Gogolins (2016) - erfolgreiche Bildungsteilhabe erlaubt.

\section{Literaturverzeichnis}

Amstein-Bahmann, C., Borgwardt, U., Brosch, M. et al. (2010). Конечно! Stuttgart: Klett.

Bachmann, T. \& Feilke, H. (Hrsg.) (2014). Werkzeuge des Schreibens. Beiträge zu einer Didaktik der Textprozeduren. Stuttgart: Fillibach Verlag.

Baumert, J., Stanat, P. \& Watermann, R. (Hrsg.) (2006). Herkunftsbedingte Disparitäten im Bildungswesen: differenzielle Bildungsprozesse und Probleme der Verteilungsgerechtfertigkeit. Vertiefende Analysen im Rahmen von PISA 2000. Wiesbaden: Springer VS.

Böhmer, J. (2015). Mehrsprachigkeit. Bd. 39: Biliteralität. Eine Studie zu literaten Strukturen in Sprachproben von Jugendlichen im Deutschen und im Russischen. Münster u. a.: Waxmann.

Anka Bergmann, Olga Caspers \& Wolfgang Stadler (Hg.)

Didaktik der slawischen Sprachen - Beiträge zum 1. Arbeitskreis in Berlin (12.-14.9.2016)

(C) 2018 innsbruck university press, ISBN 978-3-903187-11-5, DOI 10.15203/3187-11-5 
Böhmer, J. (2016). Ausprägungen von Biliteralität bei deutsch-russisch bilingualen Schülern und die daraus resultierenden Konsequenzen für den schulischen Russischunterricht. In P. Rosenberg \& C. Schroeder (Hrsg.), Mehrsprachigkeit als Ressource in der Schriftlichkeit (133-158). Berlin: de Gruyter.

Börner, W. (1992). Selbststeuerung durch Schreibmaximen im fremdsprachlichen Schreiben. In K.-H. Pogner (Hrsg.), Odense Working Papers in Language and Communication: At Skrive, Schreiben, Writing. Beiträge zur Schreibforschung und -didaktik (67-82). Odense: Odense University.

Bos, W., Hornberg, S., Arnold, K.-H., Faust, G., Fried, L., Lankes, E.-M., Schwippert, K. \& Valtin, R. (Hrsg.) (2007). IGLU 2006. Lesekompetenzen von Grundschulkindern in Deutschland im internationalen Vergleich. Münster u. a.: Waxmann.

Cummins, J. (2010). Language Support for Pupils from Families with Migration Backgrounds: Challenging Monolingual Instructional Assumptions. In C. Benholz, G. Kniffka \& E. WintersOhle (Hrsg.), Mehrsprachigkeit. Bd. 26: Fachliche und sprachliche Förderung von Schülern mit Migrationsgeschichte. Beiträge des Mercator-Symposiums im Rahmen des 15. AILA-Weltkongresses „Mehrsprachigkeit: Herausforderungen und Chancen“ (13-23). Münster u. a.: Waxmann.

Cummins, J. (1979). Linguistic Interdependence and the Educational Development of Bilingual Children. Review of Educational Research, 49 (2), 222-251.

Freie und Hansestadt Hamburg, Behörde für Schule und Berufsbildung (2011). Bildungsplan. Gymnasium. Sekundarstufe I. Neuere Fremdsprachen. Verfügbar unter: https://tinyurl.com/y9z8aqdr [02.12.2017].

Freie und Hansestadt Hamburg, Behörde für Schule und Berufsbildung (2016). Operatorenliste für die schriftlichen zentralen Abiturprüfungen 2017. Verfügbar unter: https://tinyurl.com/y7j7yksl [02.12.2017].

Gogolin, I. (2016). Zum Schluss: Optimaler HSU-Unterricht als Teil einer neuen Kultur der Sprachbildung - eine Zukunftsversion. In B. Schader (Hrsg.), Grundlagen und Hintergründe. Materialien für den herkunftssprachlichen Unterricht. Hand- und Arbeitsbuch (176-182). Zürich: Orell Füssli Verlag.

Gogolin, I. \& Lange, I. (2010). Bildungssprache und Durchgängige Sprachbildung. In S. Fürstenau \& M. Gomolla (Hrsg.), Mehrsprachigkeit (107-128). Wiesbaden: Springer VS.

Gottwald, Y. (2016). Möglichkeiten der Steuerung der Textproduktion durch Schreibmaximen im herkunftssprachlichen Russischunterricht. Unveröffentlichte Masterarbeit, Universität Hamburg. Hamburg. 
Grießhaber, W. (2001). Erst- und zweitsprachliche Textproduktion nach Bildvorlage. In A. Wolff \& E. Winters-Ohle (Hrsg.), Wie schwer ist die deutsche Sprache wirklich? (102-114). Regensburg: Fachverband Deutsch als Fremdsprache.

Grießhaber, W. (2008). Schreiben in der Zweitsprache. In B. Ahrenholz \& I. Oomen-Welke (Hrsg.), Deutschunterricht in Theorie und Praxis (Band 9: Deutsch als Zweitsprache) (228-238). Hohengehren: Schneider Verlag.

Hayes, J. R. \& Flower, L. (1980). Identifying the Organisation of Writing Processes. In L. W. Gregg \& E. R. Steinberg (Hrsg.), Cognitive Processes in Writing (3-30). Hillsdale: Lawrence Erlbaum Associates.

Kristmanson, P. L., Dicks, J. \& Le Bouthillier, J. (2009). Pedagogical Applications of a Second Language Writing Model at Elementary and Middle School Levels. Writing \& Pedagogy, 1, 37-62. Mehlhorn, G. (2016). Herkunftssprecher im Russischunterricht. Sprachliches Vorwissen als Ressource. Praxis Fremdsprachenunterricht Russisch, 5, 10-12.

Polinsky, M. \& Kagan, O. (2007). Heritage Languages: In the 'Wild' and in the Classroom. Language and Linguistics Compass, 5 (1), 368-395.

Rauch, D., Jurecka, A. \& Hesse, H.-G. (2010). Für den Drittspracherwerb zählt auch die Lesekompetenz in der Herkunftssprache. Untersuchung der Türkisch-, Deutsch- und EnglischLesekompetenz bei Deutsch-Türkisch bilingualen Schülern. Zeitschrift für Pädagogik, 56 (55. Beiheft), 78-100.

Roll, H. \& Beese, M. (2015). Textsorten im Fach. Zur Förderung von Literalität im Sachfach in der Schule und Lehrerbildung. In C. Benholz, M. Frank \& E. Gürsoy (Hrsg.), Deutsch als Zweitsprache in allen Fächern (51-72). Stuttgart: Fillibach Verlag.

Roll, H., Gürsoy, E. \& Boubakri, C. (2016). Mehrsprachige Literalität fördern. Ein Ansatz zur Koordinierung von Deutschunterricht und herkunftssprachlichem Türkischunterricht am Beispiel von Sachtexten. Der Deutschunterricht, 6, 57-67.

Second Language Research Institute of Canada (o. J). The ECRI-Model. Theoretical Foundations. Verfügbar unter: https://tinyurl.com/yclqavpz [02.12.2017].

Wolff, D. (1992). Zur Förderung der zweitsprachlichen Schreibfähigkeit. In W. Börner \& K. Vogel (Hrsg.), Schreiben in der Fremdsprache. Prozeß und Text, Lehren und Lernen (110-134). Bochum: AKS-Verlag.

Yilmaz Woerfel, S. \& Riehl, C. M. (2016). Mehrschriftlichkeit: Wechselseitige Einflüsse von Textkompetenz, Sprachbewusstheit und außersprachliche Faktoren. In P. Rosenberg \& C. Schroeder (Hrsg.), Mehrsprachigkeit als Ressource in der Schriftlichkeit (305-336). Berlin: de Gruyter.

Anka Bergmann, Olga Caspers \& Wolfgang Stadler (Hg.)

Didaktik der slawischen Sprachen - Beiträge zum 1. Arbeitskreis in Berlin (12.-14.9.2016)

(C) 2018 innsbruck university press, ISBN 978-3-903187-11-5, DOI 10.15203/3187-11-5 